ARTICLE

DOI: $10.1038 / s 41467-018-07160-7$

\title{
High-entropy high-hardness metal carbides discovered by entropy descriptors
}

\author{
Pranab Sarker ${ }^{1}$, Tyler Harrington ${ }^{2}$, Cormac Toher ${ }^{1}$, Corey Oses (iD ${ }^{1}$, Mojtaba Samiee $^{3}$, Jon-Paul Maria ${ }^{4}$, \\ Donald W. Brenner ${ }^{4}$, Kenneth S. Vecchio ${ }^{2,3} \&$ Stefano Curtarolo ${ }^{5,6}$
}

High-entropy materials have attracted considerable interest due to the combination of useful properties and promising applications. Predicting their formation remains the major hindrance to the discovery of new systems. Here we propose a descriptor-entropy forming ability - for addressing synthesizability from first principles. The formalism, based on the energy distribution spectrum of randomized calculations, captures the accessibility of equally-sampled states near the ground state and quantifies configurational disorder capable of stabilizing high-entropy homogeneous phases. The methodology is applied to disordered refractory 5-metal carbides-promising candidates for high-hardness applications. The descriptor correctly predicts the ease with which compositions can be experimentally synthesized as rock-salt high-entropy homogeneous phases, validating the ansatz, and in some cases, going beyond intuition. Several of these materials exhibit hardness up to 50\% higher than rule of mixtures estimations. The entropy descriptor method has the potential to accelerate the search for high-entropy systems by rationally combining first principles with experimental synthesis and characterization.

\footnotetext{
${ }^{1}$ Department of Mechanical Engineering and Materials Science, Duke University, Durham, NC 27708, USA. ${ }^{2}$ Materials Science and Engineering Program, University of California, San Diego, La Jolla, CA 92093, USA. ${ }^{3}$ Department of Nanoengineering, University of California, San Diego, La Jolla, CA 92093, USA. ${ }^{4}$ Department of Materials Science and Engineering, North Carolina State University, Raleigh, NC 27695, USA. ${ }^{5}$ Materials Science, Electrical Engineering, Physics and Chemistry, Duke University, Durham, NC 27708, USA. ${ }^{6}$ Fritz-Haber-Institut der Max-Planck-Gesellschaft, 14195 Berlin-Dahlem, Germany. These authors contributed equally: Pranab Sarker, Tyler Harrington. Correspondence and requests for materials should be addressed to K.S.V. (email: kvecchio@eng.ucsd.edu) or to S.C. (email: stefano@duke.edu)
} 
igh-entropy materials having a highly disordered homogeneous crystalline single phase (potentially stabilized entirely by entropic contributions) continue to attract a great deal of research interest ${ }^{1-3}$. Remarkable properties have been reported: high strength (yield stress $>1 \mathrm{GPa}$ ) combined with ductility ${ }^{4-11}$, hardness ${ }^{5,12,13}$, superconductivity ${ }^{14}$, colossal dielectric constant ${ }^{15}$, and superionic conductivity ${ }^{16}$. Entropy is thought to play a key-stabilizing role in high-entropy alloys ${ }^{1}$, entropy-stabilized oxides ${ }^{17,18}$, high-entropy borides ${ }^{19}$, and highentropy carbides ${ }^{20-23}$. The latter three classes consist of disordered metal cation sublattices with several species at equiconcentration combined with oxide ${ }^{15,17,18,24}$, boride ${ }^{19}$, or carbide $^{20-23}$ anion sublattices. These systems offer the potential to combine excellent thermo-mechanical properties and resilient thermodynamic stability given by entropy stabilization with the higher oxidation resistance of ceramics ${ }^{25}$. The resistance of disordered carbides to extreme heat ${ }^{26-28}$, oxidation ${ }^{23}$, and wear makes them promising ultra-high-temperature ceramics for thermal protection coatings in aerospace applications ${ }^{29}$, and as high-hardness, relatively low-density high-performance drill bits and cutting tools in mining and industry.

Super-hard transition metal carbides have been known since the 1930s to exhibit significant levels of solid solution ${ }^{26,30,31}$, and to display high melting temperatures ${ }^{26,27}$. $\mathrm{Ta}_{x} \mathrm{Hf}_{1-x} \mathrm{C}$ forms a homogeneous solid solution across all composition ranges ${ }^{28,32,33}$, with $\mathrm{Ta}_{4} \mathrm{HfC}_{5}$ exhibiting one of the highest experimentally reported melting points $\left(T_{\mathrm{m}} \sim 4263 \mathrm{~K}^{26,27}\right)$. In this case, the two refractory metals randomly populate one of the two rock-salt sublattices $^{28}$. More recent measurements indicate that the maximum melting point of $4232 \mathrm{~K}$ occurs without $\mathrm{Ta}$ at the composition $\mathrm{HfC}_{0.98}{ }^{34}$. Investigation of new carbide compositions will help elucidate the high temperature behavior of these materials, and will provide an avenue to settle the discrepancies in the experimental literature. To discover materials with even more advantageous properties, including increased thermal stability, enhanced strength and hardness, and improved oxidation resistance ${ }^{23}$, more species and configurations have to be considered. Unfortunately, the lack of a rational, effective, and rapid method to find and characterize the disordered crystalline phase makes it impossible to pinpoint the right combination of species/compositions and the discovery continues by slow and relatively expensive trial and error.

Computationally, the hindrance in in-silico disordered materials development can be attributed to entropy-a very difficult quantity to parameterize when searching through the immense space of candidates (even with efficient computational methods, e.g., Monte Carlo and ab-initio lattice energies in the WangLandau $^{35}$ or nested sampling ${ }^{36}$ formalisms). CALPHAD has also been applied successfully $2,13,37-39$, although it is dependent on the availability of sufficient experimental data. This is the perfect challenge for ab-initio high-throughput computing 40 as long as reasonable entropy descriptors-the set of parameters capturing the underlying mechanism of a materials property_can be found.

In this article, we undertake the challenge by formulating an entropy-forming-ability (EFA) descriptor. It captures the relative propensity of a material to form a high-entropy single-phase crystal by measuring the energy distribution (spectrum) of configurationally randomized calculations up to a given unit-cell size. A narrow spectrum implies a low energy cost for accessing metastable configurations, hence promoting randomness (i.e., entropy) into the system (high-EFA) at finite temperature. In contrast, a wide spectrum suggests a composition with a high energy barrier for introducing different configurations (low-EFA), and thus with a strong preference for ordered phases. The method is benchmarked by the matrix of possible carbides. Given a set of eight refractory metals ( $\mathrm{Hf}, \mathrm{Nb}, \mathrm{Mo}, \mathrm{Ta}, \mathrm{Ti}, \mathrm{V}, \mathrm{W}$, and $\mathrm{Zr}$ ) plus carbon, the formalism predicts the matrix of synthesizable fivemetal high-entropy carbides. Candidates are then experimentally prepared, leading to a novel class of systems. In particular, it is demonstrated that the descriptor is capable of reliably distinguishing between the compositions that easily form homogeneous single phases and the ones that do not, including identifying compositions that form single phases despite incorporating multiple binary carbide precursors with different structures and stoichiometric ratios. Note that because of the differing stoichiometries of the non-rock-salt phase binary carbide precursors $\mathrm{Mo}_{2} \mathrm{C}$ and $\mathrm{W}_{2} \mathrm{C}$, the compositions listed in this work are nominal. There are extensive carbon vacancies in the anion sublattice in the synthesized materials, further contributing to the configurational entropy. Several of these materials display enhanced mechanical properties, e.g., Vickers hardness up to $50 \%$ higher than predicted by a rule of mixtures (ROM). Thus, this class of materials has strong potential for industrial uses where dense and wearresistant impactors are needed, particularly for extreme temperature applications. The successful outcome demonstrates the strength of the synergy between thermodynamics, highthroughput computation, and experimental synthesis.

\section{Results}

EFA formalism. To accelerate the search in the chemical space, the entropy content of a compound is estimated from the energy distribution spectrum of metastable configurations above the zero-temperature ground state. At finite $T$, any disordered state can be present with a probability given by the Boltzmann distribution and the state's degeneracy. Note that configurations are randomly sampled up to a given unit-cell size: the larger the size, the more accurately the spectrum represents the real thermodynamic density of states.

The energy distribution $\left(H_{i}\right)$ spectrum can be quantitatively characterized by its standard deviation $\sigma$, so that the $\sigma$ becomes the descriptor for $S$ : the smaller $\sigma$, the larger $S$. The descriptor for an $\mathrm{N}$-species system, called the EFA, is defined as the inverse of the $\sigma$ of the energy spectrum above the ground state of the $\mathrm{N}$-system at zero temperature:

$$
\operatorname{EFA}(N) \equiv\left\{\sigma\left[\operatorname{spectrum}\left(H_{i}(N)\right)\right]_{T=0}\right\}^{-1}
$$

where

$$
\sigma\left\{H_{i}(N)\right\}=\sqrt{\frac{\sum_{i=1}^{n} g_{i}\left(H_{i}-H_{\text {mix }}\right)^{2}}{\left(\sum_{i=1}^{n} g_{i}\right)-1}},
$$

where $n$ is the total number of sampled geometrical configurations and $g_{i}$ are their degeneracies. $H_{\mathrm{mix}}$ is the mixed-phase enthalpy approximated by averaging the enthalpies $H_{i}$ of the sampled configurations:

$$
H_{\text {mix }}=\frac{\sum_{i=1}^{n} g_{i} H_{i}}{\sum_{i=1}^{n} g_{i}} .
$$

The broader the spectrum, the more energetically expensive it will be to introduce configurational disorder into the system, and thus the lower the EFA. EFA is measured in (eV/atom) $)^{-1}$.

EFA calculation. A total of 56 five-metal compositions can be generated using the eight refractory metals $(8 ! / 5 ! 3 !=56)$ of interest (Hf, Nb, Mo, Ta, Ti, V, W, and $\mathrm{Zr}$ ). For each 
composition, the Hermite normal form superlattices of the Automatic FLOW (AFLOW) partial occupation (AFLOWPOCC) method $^{41}$ generate 49 distinct 10 -atom-cell configurations, resulting in a total of 2744 configurations needed to determine the EFA of this composition space (Methods section). The ab-initio calculated EFA values for the full set of 56 fivemetal compositions are provided in Table 1. Nine candidates are chosen from this list for experimental synthesis and investigation: (i) the three candidates with the highest value of EFA (MoN$\mathrm{bTaVWC}_{5}\left(\mathrm{EFA}=125(\mathrm{eV} / \text { atom })^{-1}\right), \mathrm{HfNbTaTiZrC}_{5}(\mathrm{EFA}=$ $\left.100(\mathrm{eV} / \text { atom })^{-1}\right), \mathrm{HfNbTaTiVC}_{5}\left(\mathrm{EFA}=100(\mathrm{eV} / \text { atom })^{-1}\right)$, high probability of forming high-entropy single phases), (ii) the two candidates with the lowest value of EFA ( $\mathrm{HfMoVWZrC}_{5}(37$ $\left.(\mathrm{eV} / \text { atom })^{-1}\right), \mathrm{HfMoTiWZrC}_{5}\left(38(\mathrm{eV} / \text { atom })^{-1}\right)$, low probability of forming high-entropy single phases), and (iii) four chosen at random with intermediate $\mathrm{EFA}\left(\mathrm{NbTaTiVWC}_{5}\right.$ (77 (eV/atom) $\left.\left.{ }^{-1}\right), \operatorname{HfNbTaTiWC}_{5}(67 \text { (eV/atom })^{-1}\right), \mathrm{HfTaTiWZrC}_{5}(50(\mathrm{eV} /$ atom $)^{-1}$ ), and $\mathrm{HfMoTaWZrC}_{5}\left(45(\mathrm{eV} / \text { atom })^{-1}\right)$ ). Figure la shows the energy distribution and EFA values obtained ab initio from configurations generated with AFLOW for the nine chosen systems. For $\mathrm{MoNbTaVWC}_{5}, \mathrm{HfNbTaTiZrC}_{5}$, and HfNbTa$\mathrm{TiVC}_{5}$, most of the configurations are within $20 \mathrm{meV} /$ atom of the lowest energy state, and the distributions have an EFA of at least $100(\mathrm{eV} / \mathrm{atom})^{-1}$. Therefore, at finite temperature, most of the configurations should have a high probability of being formed, so that a high level of configurational randomness is expected to be accessible in the three systems, making them promising candidates to form a high-entropy homogeneous single phase. Achieving a similar level of configurational randomness would be progressively more difficult in $\mathrm{NbTaTiVWC}_{5}, \mathrm{HfNbTaTiWC}_{5}$, and $\mathrm{HfTaTiWZrC}_{5}$ as the different configurations display a broader energy distribution, with EFAs ranging from 50 to 77 (eV/atom $)^{-1}$. A higher energy cost is needed to incorporate configurational entropy into these three compositions, so forming a homogeneous single phase will be more difficult. For HfMo$\mathrm{TaWZrC}_{5}, \mathrm{HfMoTiWZrC}_{5}$, and $\mathrm{HfMoVWZrC}_{5}$, the spread of energies for the configurations is very wide, with EFA values from 45 down to $37(\mathrm{eV} / \mathrm{atom})^{-1}$. These materials would be expected to be very difficult to synthesize as a homogeneous single phase.

Competing ordered phases. The phase diagrams for the fivemetal carbide systems were generated to investigate the existence of binary and ternary ordered structures that could compete with the formation of the high-entropy single phase. First, prototypes for experimentally reported binary and ternary carbide structures $^{42,43}$ are used to calculate the formation enthalpies of additional ordered phases for the AFLOW database. The results are used to generate the convex hull phase diagrams for all 56 compositions using the AFLOW-CHULL module ${ }^{44}$. The relevant binary and ternary convex hulls are illustrated in Supplementary Figures 1-36. The distance along the enthalpy axis of the lowest energy AFLOW-POCC configuration from the convex hull, $\Delta H_{\mathrm{f}}$, is listed in Table 1 (the decomposition reaction products are summarized in Supplementary Table 2). A rough estimation of the synthesis temperature $T_{\mathrm{s}}$ (see the analogous EntropyStabilized Oxides case, Fig. 2 of ref. ${ }^{17}$ ) - can be calculated by dividing $\Delta H_{\mathrm{f}}$ by the ideal configuration entropy (Supplementary Table 1 , with the ideal entropy per atom evaluated as $0.5 k_{\mathrm{B}} \times$ $\log 0.2$, since $k_{\mathrm{B}} \times \log 0.2$ is the entropy per metal carbide atomic pair). A precise characterization of the disorder requires more expensive approaches, such as the LTVC method ${ }^{45}$, and is beyond the scope of this article. The highest temperature is $2254 \mathrm{~K}$, which is less than the synthesis temperature of $2200^{\circ} \mathrm{C}(2473 \mathrm{~K})$, indicating that during sintering the disordered phases are thermodynamically accessible with respect to decomposition into ordered compounds. In analogy to the formation of metallic glasses where energetic confusion obstructs crystalline growth $^{46,47}$ once the temperature is reduced, systems with high EFA remain locked into ensembles of highly degenerate configurations, retaining the disorder achieved at high temperature. Hence, EFA provides a measure of the relative synthesizability of the disordered composition.

From enthalpy to entropy. It is important to consider that the metal carbide precursors have very strong covalent/ionic bonds, and are therefore enthalpy stabilized ${ }^{48}$. However, the same might not be the case for their mixture. In fact, a statistical analysis of the AFLOW.org enthalpies ${ }^{44}$ indicates that the gain in formation enthalpy by adding mixing species, $\Delta H_{\mathrm{f}}(N+1)-\Delta H_{\mathrm{f}}(N)$, decreases with $N$, and can easily be overcome by the monotonic increase in entropy-gain for the disordered systems (to go from order to complete or partial disorder). In the AFLOW analysis, the threshold between low- and high-entropy systems is around four mixing species. To have completely entropy-stabilized

Table 1 Results for the calculated entropy-forming-ability (EFA) descriptor, energetic distance from six-dimensional convex hull $\left(\Delta H_{f}\right)$ and vibrational free energy at $2000 \mathrm{~K}\left(\Delta F_{\mathrm{vib}}\right)$ for the five-metal carbide systems, arranged in descending order of EFA

\begin{tabular}{|c|c|c|c|c|c|c|c|c|c|c|c|c|c|c|c|c|c|}
\hline System & EFA & $\Delta H_{f}$ & $\Delta F_{\text {vib }}$ & Exp. & $\varepsilon$ & System & EFA & $\Delta H_{\mathrm{f}}$ & $\Delta F_{\text {vib }}$ & Exp. & $\varepsilon$ & System & EFA & $\Delta H_{\mathrm{f}}$ & $\Delta F_{\text {vib }}$ & Exp. & $\varepsilon$ \\
\hline MoNbTaVWC $_{5}$ & 125 & 156 & -14 & $\mathrm{~S}$ & 0.063 & $\mathrm{HfNbTaVWC}_{5}$ & 67 & 110 & & & & $\mathrm{TaTiVWZrC}_{5}$ & 50 & 96 & & & \\
\hline $\mathrm{HfNbTaTiZrC}_{5}$ & 100 & 19 & -12 & $\mathrm{~S}$ & 0.094 & HfMoTaTiVC $_{5}$ & 67 & 82 & & & & $\mathrm{NbTiVWZrC}_{5}$ & 50 & 93 & & & \\
\hline $\mathrm{HfNbTaTiVC}_{5}$ & 100 & 56 & -31 & $\mathrm{~S}$ & 0.107 & $\mathrm{HfMoNbTiZrC}_{5}$ & 67 & 53 & & & & $\mathrm{HfMoTiVZrC}_{5}$ & 50 & 96 & & & \\
\hline MoNbTaTiVC $_{5}$ & 100 & 82 & & & & MoNbTaWZrC $_{5}$ & 63 & 133 & & & & HfMoTaVZrC 5 & 50 & 92 & & & \\
\hline $\mathrm{NbTaTiVZrC}_{5}$ & 83 & 64 & & & & HfMoTaTiZrC 5 & 63 & 55 & & & & $\mathrm{HfMoNbVZrC}_{5}$ & 50 & 89 & & & \\
\hline $\mathrm{HfMoNbTaTiC}_{5}$ & 83 & 48 & & & & $\mathrm{NbTaTiWZrC}_{5}$ & 59 & 61 & & & & MoTaVWZrC 5 & 48 & 148 & & & \\
\hline $\mathrm{NbTaTiVWC}_{5}$ & 77 & 92 & -19 & $\mathrm{~S}$ & 0.124 & MoTaTiVZrC 5 & 59 & 92 & & & & MoTaTiWZrC 5 & 48 & 94 & & & \\
\hline MoNbTaTiWC $_{5}$ & 77 & 111 & & & & MoNbTiVZrC 5 & 59 & 87 & & & & MoNbVWZrC 5 & 48 & 146 & & & \\
\hline MoNbTiVWC $_{5}$ & 71 & 122 & & & & MoNbTaVZrC 5 & 59 & 108 & & & & MoNbTiWZrC 5 & 48 & 89 & & & \\
\hline MoNbTaTiZrC $_{5}$ & 71 & 57 & & & & $\mathrm{HfNbTiVWC}_{5}$ & 59 & 81 & & & & $\mathrm{HfMoNbWZrC}_{5}$ & 48 & 101 & & & \\
\hline HfTaTiVZrC $_{5}$ & 71 & 73 & & & & $\mathrm{HfNbTaWZrC}_{5}$ & 59 & 53 & & & & $\mathrm{HfTiVWZrC}_{5}$ & 45 & 99 & & & \\
\hline $\mathrm{HfNbTiVZrC}_{5}$ & 71 & 73 & & & & $\mathrm{NbTaVWZrC}_{5}$ & 56 & 119 & & & & $\mathrm{HfNbVWZrC}_{5}$ & 45 & 94 & & & \\
\hline $\mathrm{HfMoNbTiVC}_{5}$ & 71 & 77 & & & & $\mathrm{HfTaTiVWC}_{5}$ & 56 & 84 & & & & $\mathrm{HfMoTiVWC}_{5}$ & 45 & 97 & & & \\
\hline $\mathrm{HfMoNbTaZrC}_{5}$ & 71 & 48 & & & & HfMoTaVWC $_{5}$ & 56 & 139 & & & & HfMoTaWZrC ${ }_{5}$ & 45 & 105 & & M & 0.271 \\
\hline $\mathrm{HfMoNbTaWC}_{5}$ & 71 & 126 & & & & $\mathrm{HfMoNbVWC}_{5}$ & 56 & 137 & & & & $\mathrm{HfTaVWZrC}_{5}$ & 43 & 97 & & & \\
\hline $\mathrm{HfMoNbTaVC}_{5}$ & 71 & 99 & & & & $\mathrm{HfNbTiWZrC}_{5}$ & 53 & 56 & & & & MoTiVWZrC 5 & 40 & 107 & & & \\
\hline HfNbTaTiWC $_{5}$ & 67 & 53 & $\sim 0$ & $\mathrm{~S}$ & 0.171 & HfMoTaTiWC $_{5}$ & 53 & 84 & & & & HfMoTiWZrC $_{5}$ & 38 & 83 & & M & 0.315 \\
\hline MoTaTiVWC $_{5}$ & 67 & 128 & & & & $\mathrm{HfMoNbTiWC}_{5}$ & 53 & 81 & & & & HfMoVWZrC 5 & 37 & 141 & & M & 0.325 \\
\hline $\mathrm{HfNbTaVZrC}_{5}$ & 67 & 60 & & & & $\mathrm{HfTaTiWZrC}_{5}$ & 50 & 59 & $\sim 0$ & $\mathrm{~S}$ & 0.169 & & & & & & \\
\hline
\end{tabular}

Nine compositions are selected for experimental investigation. The lattice distortion, $\varepsilon$, is obtained from the peak broadening in XRD. S: single-phase formed; $M$ : multi-phase formed in experiment. Note that the compositions listed here are nominal, and the actual synthesized compositions can vary due to the presence of carbon vacancies in the anion sublattice. Units: $E F A$ in (eV/atom) ${ }^{-1} ; \Delta H_{\mathrm{f}}$ and $\Delta F_{\text {vib }}$ in (meV/atom); and $\varepsilon$ in \% 
a



b

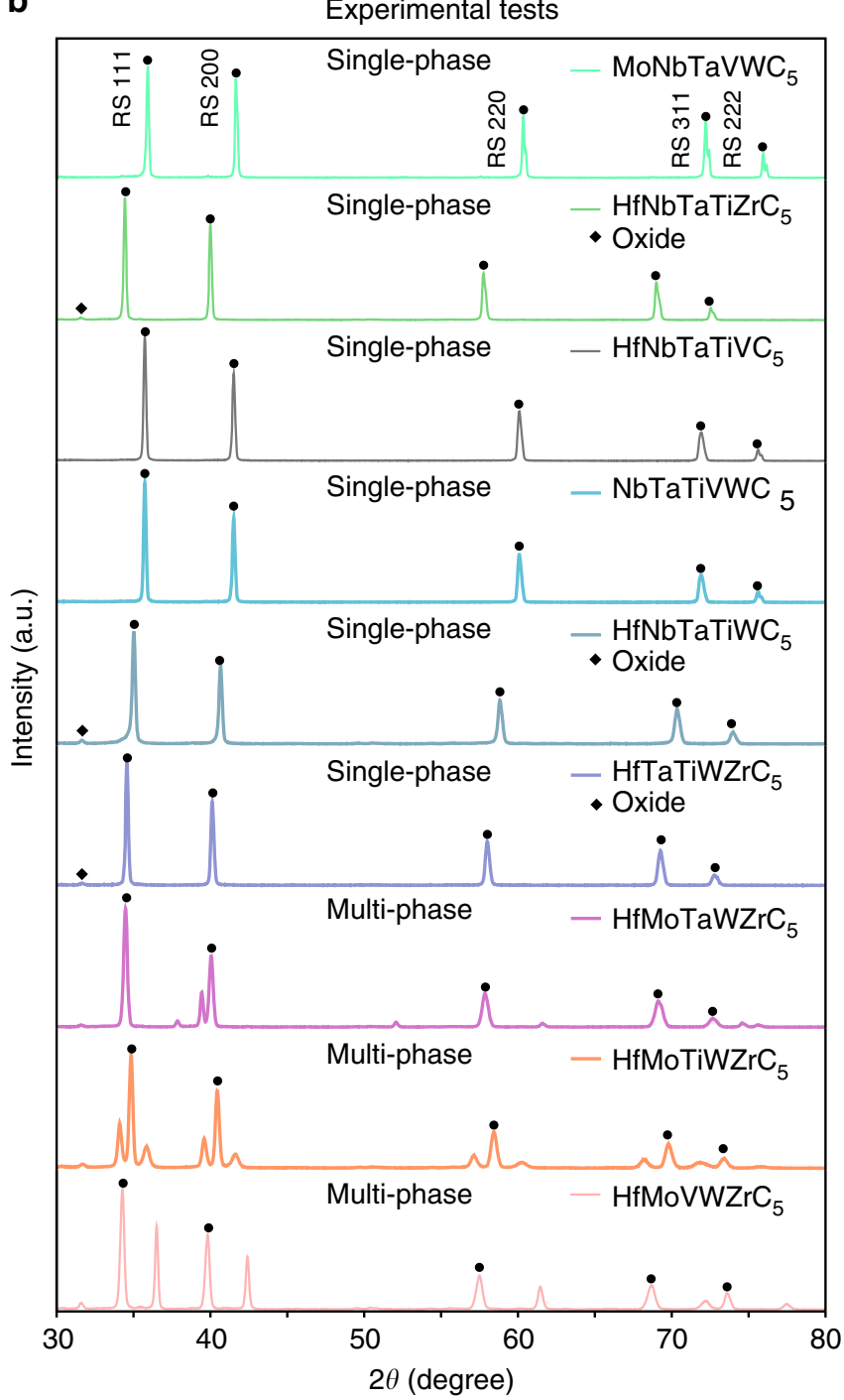

Fig. 1 Schematics of high-entropy carbides predictions. a The energy distribution of different configurations of the 9 five-metal carbides: MoNbTaVWC ${ }_{5}$, $\mathrm{HfNbTaTiZrC}_{5}, \mathrm{HfNbTaTiVC}_{5}, \mathrm{NbTaTiVWC}_{5}, \mathrm{HfNbTaTiWC}_{5}, \mathrm{HfTaTiWZrC}_{5}, \mathrm{HfMoTaWZrC}_{5}, \mathrm{HfMoTiWZrC}_{5}$, and HfMoVWZrC H $_{\text {; }}$ spectrum is shifted so that the lowest energy configuration for each composition is at zero. The energy spectrum for each composition indicates its propensity to form the high-entropy single phase: the narrower the distribution, the more likely it is to form a high-entropy single phase at finite $T$. $\mathbf{b}$ The $\mathrm{X}$-ray diffraction patterns for the same 9 five-metal carbides, where the first six compositions exhibit only the desired fcc structure peaks, whereas the additional peaks for remaining three compositions indicate the presence of secondary phases. The small peaks at $2 \theta=31.7^{\circ}$, marked by the diamond symbol in the spectra of $\mathrm{HfNbTaTiZrC}_{5}, \mathrm{HfNbTaTiWC}_{5}$ and $\mathrm{HfTaTiWZrC}_{5}$, are from the (111) plane of a monoclinic $(\mathrm{Hf}, \mathrm{Zr}) \mathrm{O}_{2}$ phase that remains due to processing

materials, five mixing species are required (similar to the Entropy-Stabilized Oxides ${ }^{17}$ ). For carbides in which only the metal-sublattice is randomly populated, five metals should be enough to achieve carbide entropy stabilization, especially at equi-composition. Notably, if other sublattices were also allowed to contain disorder, (e.g., reciprocal systems like $\mathrm{Ta}_{x} \mathrm{Hf}_{1-x} \mathrm{C}_{1}$ $-y^{26,27}$ ), then the overall number of species might reduce. In this example, entropy was increased by introducing point defects (vacancies) - a promising strategy to improve high temperature performance. In $\mathrm{HfC}_{1-x}$, the reduction of $\mathrm{C}$ to sub-stoichiometry enhances the stabilizing effect of the configurational entropy on the solid phase, offsetting its Gibbs free energy (vacancies can only exist in the solid phase) leading to an overall increase in melting point (see ref. ${ }^{28}$ ).

Experimental results. To validate the predictions, the nine chosen carbides are experimentally synthesized and characterized (see Methods section). The chemical homogeneity of each sample is measured using energy-dispersive X-ray spectroscopy (EDS), whereas the crystalline structure is determined via X-ray diffraction (XRD).

An example of the evolution of a sample of composition $\mathrm{HfNbTaTiVC}_{5}$ through each processing step is given in Fig. 2a, demonstrating the densification and homogenization into a single rock-salt structure. At least three distinct precursor phases are distinguishable in the mixed powder pattern. Following ball milling, the individual phases are still present, however, the peaks are considerably broadened, which is due to particle size reduction and mechanical alloying. Following the final spark plasma sintering (SPS) step at $2200^{\circ} \mathrm{C}$, the sample consolidates into a bulk solid pellet of the desired single rock-salt phase indicating the successful synthesis of a high-entropy homogeneous carbide.

Results of XRD analysis for each sample following SPS at 2200 ${ }^{\circ} \mathrm{C}$, presented in Fig. $1 \mathrm{~b}$, demonstrate that compositions $\mathrm{MoN}$ bTaVWC $_{5}$, HfNbTaTiZrC $_{5}$, HfNbTaTiVC $_{5}$, HfNbTaTiWC $_{5}$, $\mathrm{NbTaTiVWC}_{5}$, and HfTaTiWZrC $\mathrm{H}_{5}$ (the top 6) only exhibit single 

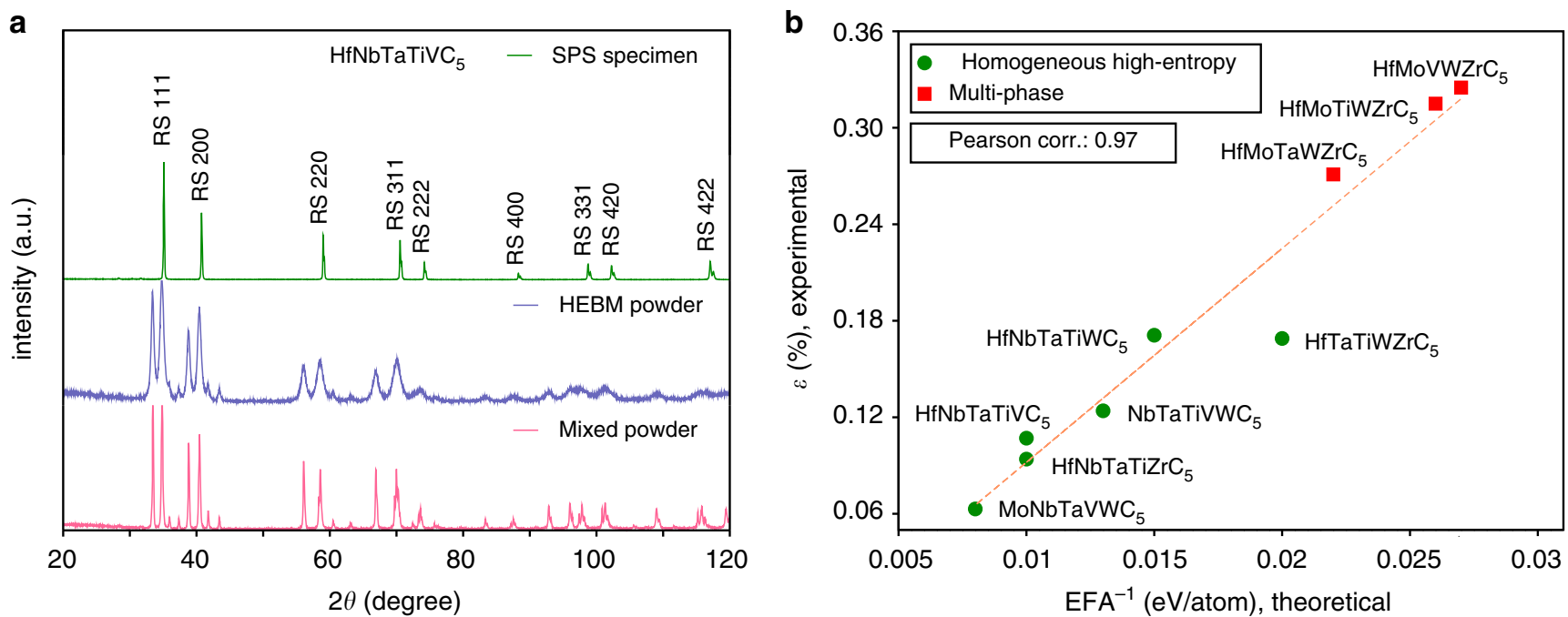

C

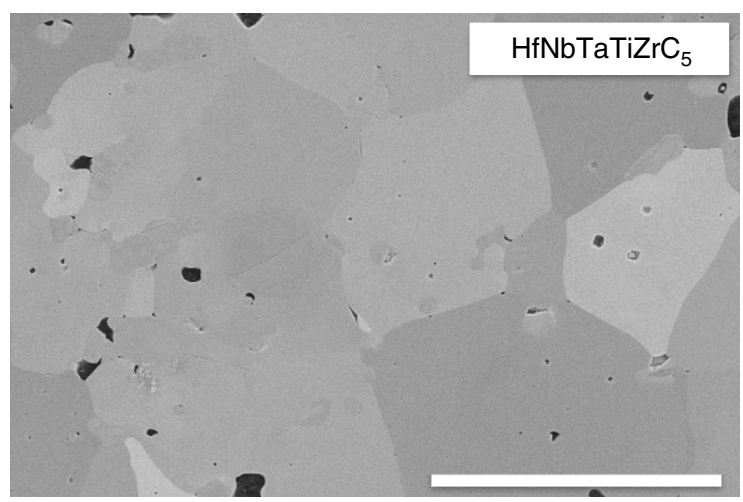

d

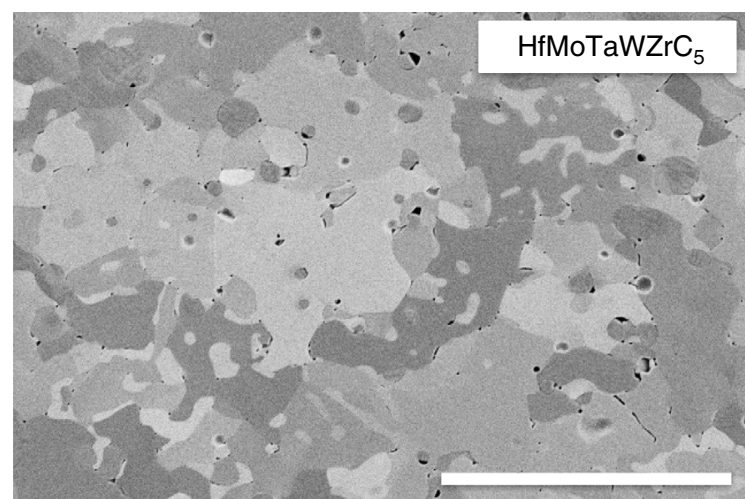

e
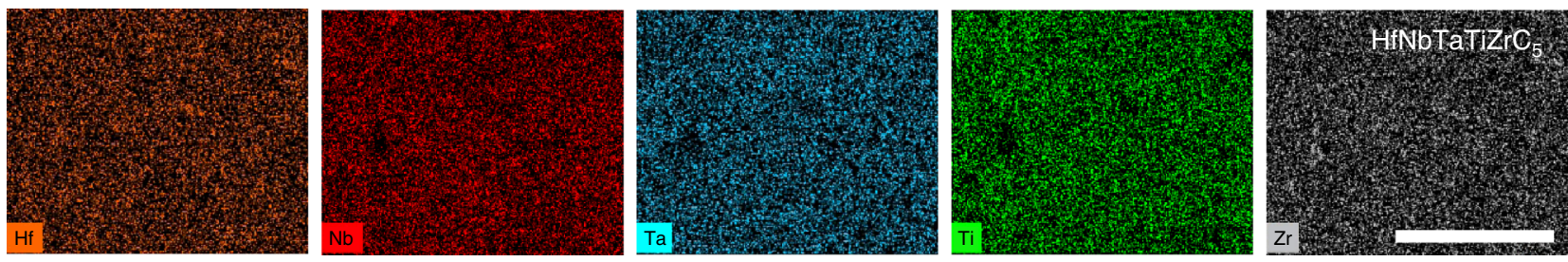

$\mathbf{f}$


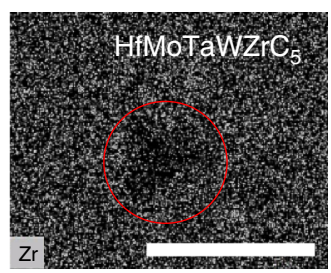

Fig. 2 Experimental results for high-entropy carbides synthesis and characterization. a Progression of a sample of HfNbTaTiVC $\mathrm{H}_{5}$ through each processing step: hand mixing (magenta spectrum, bottom), ball milling (blue spectrum, center), and spark plasma sintering (green spectrum, top), depicting the evolution towards the desired rock-salt crystal structure $\left(a_{\exp }=4.42 \AA\right)$. b Linear relationship between EFA ${ }^{-1}$ and the distortion of experimental lattice parameters $\varepsilon$. Green circles and red squares indicate homogeneous high-entropy single- and multi-phase compounds, respectively. c, $\mathbf{d}$ Electron micrographs of single-phase $\mathrm{HfNbTaTiZrC}_{5}$ and multi-phase $\mathrm{HfMoTaWZrC}_{5}$ specimens. e, $\mathbf{f}$ Selected EDS compositional maps of the HfNbTaTiZrC $\mathrm{C}_{5}$ and HfMoTaWZrC 5 specimens. The micrographs show the presence of the secondary phase in $\mathrm{HfMoTaWZrC}_{5}$ (circles) that is also present in XRD results, which is revealed to be a $\mathrm{W}$ - and Mo-rich phase. Scale bars, $10 \mu \mathrm{m}(\mathbf{c}-\mathbf{f})$

fcc peaks of the desired high-entropy phase (rock-salt), whereas HfMoTaWZrC 5 , HfMoTiWZrC ${ }_{5}$, and $\mathrm{HfMoVWZrC}_{5}$ (the bottom 3) show multiple structures. The small peaks at $2 \theta=31.7^{\circ}$, marked by the diamond symbol in the spectra of $\mathrm{HfNbTaTiZrC}_{5}$, $\mathrm{HfNbTaTiWC}_{5}$, and HfTaTiWZrC $\mathrm{C}_{5}$ in Fig. 1b, are from the (111) plane of a monoclinic ( $\mathrm{Hf}, \mathrm{Zr}) \mathrm{O}_{2}$ phase that remains due to processing. The volume fraction of this phase is $<5 \%$ and does not significantly alter the composition of the carbide phase. The distinguishable second phase in HfMoTaWZrC $\mathrm{C}_{5}$ is identified as a hexagonal phase. One and two secondary fcc phases are observed for $\mathrm{HfMoVWZrC}_{5}$ and $\mathrm{HfMoTiWZrC}_{5}$, respectively. Microstructure analysis and selected elemental mapping (Figs. 2c-d) confirm that the systems displaying single phases are chemically homogeneous, whereas the multi-phase samples undergo chemical 
segregation. For example, only grain orientation contrast is present in the $\mathrm{HfNbTaTiZrC}$ microstructure, and no indication of notable clustering or segregation is visible in its compositional maps. On the contrary, a clear chemical phase contrast is observable in the microstructure of the multi-phase $\mathrm{HfMoTaWZrC}_{5}$ sample, and the compositional maps demonstrate that the secondary phase, apparent in XRD, is $\mathrm{W}$ - and Mo-rich.

Homogeneity analysis. Peak broadening in XRD patterns (Fig. 1b) is used to quantify the level of structural homogenization achieved in the samples. According to the Williamson-Hall formulation ${ }^{49}$, broadening in XRD is principally due to crystallite size $(\propto 1 / \cos \theta, \theta=$ Bragg angle) and lattice strain $(\propto 1 / \tan \theta)$. For multi-component systems, significant broadening is expected to occur due to local lattice strains and variations in the interplanar spacings throughout the sample ${ }^{50}$. The latter can be attributed to the inhomogeneous distribution of the elements, the extent of which can be evaluated by applying the analysis to a multicomponent system, which has only a single lattice structure and is assumed strain free ${ }^{50}$.

The lattice distortion of the rock-salt phase in the single-phase materials (or the most prevalent in the multi-phase ones) is determined by using the relationship between broadening $\beta_{\mathrm{S}}$ and Bragg angle $\theta$ (Methods section):

$$
\beta_{\mathrm{S}} \cos \theta=4 \varepsilon \sin \theta+\frac{K \lambda}{D},
$$

where $\varepsilon$ is the lattice strain or variation in interplanar spacing due to chemical inhomogeneity, $K$ is a constant (dependent on the grain shape), $\lambda$ is the incident $\mathrm{X}$-ray wavelength, and $D$ is the crystallite size. Since materials are assumed strain free, $\varepsilon-$ obtained by inverting Eq. 4-represents the relative variation of the lattice parameter due to inhomogeneity. Thus, $\varepsilon$ is both a measure of homogeneity and of the effective mixing with respect to the ideal scenario. The results for the EFA descriptor, the experimental characterization, as well as the values for $\varepsilon$ for all nine carbides compositions are given in Table 1 . The values for $\varepsilon$ range from $0.063 \%$ for $\mathrm{MoNbTaVWC}_{5}$ and $0.094 \%$ for $\mathrm{HfNbTaTiZrC}_{5}$ (the most homogeneous materials) to $0.325 \%$ for HfMoVWZrC $\mathrm{H}_{5}$ (the least homogeneous material). Overall, the experimental findings agree well with the predictions of EFA descriptor, validate its ansatz and indicate a potential threshold for our model of five-metal carbides: EFA $\sim 50(\mathrm{eV} / \text { atom })^{-1} \Rightarrow$ homogeneous disordered single phase (high entropy).

High-entropy synthesizability. The comparison between the EFA predictions and the homogeneity of the samples is analyzed in Fig. 2b. Although the Williamson-Hall formalism does not provide particularly accurate absolute values of $\varepsilon$, it is effective for comparing similarly processed samples, determining the relative homogeneity. The lattice distortion $\varepsilon$ (capturing homogeneity) decreases linearly with the increase of EFA. The Pearson (linear) correlation of $\mathrm{EFA}^{-1}$ with $\varepsilon$ is 0.97 , whereas the Spearman (rank order) correlation is 0.98 . As such, the EFA takes the role of an effective high-entropy synthesizability descriptor.

Three facts are relevant. (i) Intuitively, several of the carbide compositions that easily form a highly homogeneous phase, particularly $\mathrm{HfNbTaTiVC}_{5}$ and $\mathrm{HfNbTaTiZrC}_{5}$, come from binary precursors having the same structure and ratio of anions to cations as the final high-entropy material. (ii) Counterintuitively, the highest-EFA and most homogeneous phase $\mathrm{MoNbTaVWC}_{5}$ is made with two precursors having different structures and stoichiometric ratios from the high-entropy material, specifically orthorhombic $\alpha-\mathrm{Mo}_{2} \mathrm{C}$ and hexagonal $\alpha$ $\mathrm{W}_{2} \mathrm{C}$, leading to a final sub-stoichiometric MoNbTaVWC $\mathrm{C}_{5-x}$.
The additional disorder provided by the presence of vacancies in the C-sublattice is advantageous: it allows further entropy stabilization, potentially increasing the melting point vis-à-vis the stoichiometric composition ${ }^{28}$. An investigation of the effect of carbon stoichiometry is clearly warranted, although it is outside of the scope of this study. (iii) For tungsten and molybdenum, metal-rich carbides are used because of the difficulties in obtaining molybdenum monocarbide (MoC) powder, or tungsten monocarbide (WC) powder in the particle sizes compatible with the other precursors, hindering consistent mixing, sintering and homogenization. It should be noted that additional samples of $\mathrm{MoNbTaVWC}_{5}$ were also synthesized using hexagonal WC with a smaller particle size, and the homogeneous rock-salt phase was again successfully obtained (see Supplementary Figure 37). The existence of phase-pure MoNbTaVWC $\mathrm{M}_{5}$ indicates that, similar to the rock-salt binary carbides, the multi-component carbide is stable over a range of stoichiometry. From experimental/ phenomenological grounds, the formation of such a phase is surprising. The equi-composition binary carbides $\mathrm{MoC}$ and $\mathrm{WC}$ have hexagonal ground states, and their rock-salt configurations have significantly higher formation enthalpy ${ }^{51}$. Considering these facts, there are no experimental indications that adding Mo and $\mathrm{W}$ would contribute to stabilizing the most homogeneous rocksalt five-metal carbide that was predicted by the EFA and later validated experimentally. The arguments demonstrate the advantage of a descriptor that quantifies the relative EFA over simple empirical/phenomenological rules, in that it correctly identifies this composition as having a high propensity to form a single phase, while simultaneously correctly predicting that several other systems having both Mo and W subcomponents undergo phase separation.

Mechanical properties. The Vickers hardness, $H_{\mathrm{V}}$, and elastic modulus, $E$, of both the binary carbide precursors and the synthesized five-metal single-phase compositions are measured using nanoindentation, where the properties are extracted from loaddisplacement curves (see Supplementary Figure 38(a)). The binary precursor samples for these measurements are prepared and analyzed using the same protocol to ensure the validity of the comparisons (see Methods section for more details). The results for the five-metal compositions are in Table 2, whereas those for the binary carbides are listed in Table 3 . It is found that, for the five-metal compositions, the measured $H_{\mathrm{V}}$ and $E$ values exceed those predicted from a ROM based on the binary precursor measurements. The enhancement of the mechanical properties is particularly strong in the case of $\mathrm{HfNbTaTiZrC}_{5}$, where the measured $E$ and $H_{\mathrm{V}}$ exceed the ROM predictions by 10 and $50 \%$, respectively (see Supplementary Figure 38(b) for a comparison between the $H_{\mathrm{V}}$ results obtained from calculation, experiment, and ROM). Mass disorder is one possible source of the enhanced hardness: deformation is caused by dislocation movements and activation energy is absorbed and released at each lattice step. An ideal ordered system can be seen as a dislocation-wave-guide with matched (uniform) impedance along the path: propagation occurs without any relevant energy reflection and/or dispersion. This is not the case for disordered systems: mass inhomogeneity causes impedance mismatch, generating reflections and disturbing the transmission by dispersing (scattering) its group energy. Macroscopically the effect is seen as increased resistance to plastic deformations-more mechanical work is required-i.e., increase of hardness. Other possible causes of increased hardness include solid solution hardening $5,13,20$, where the atomic size mismatch results in lattice distortions, limiting the motion of dislocations necessary for plastic deformation; and changes in the slip systems and the ease with which slip can occur 20,52 . 


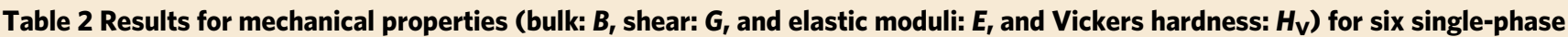
high-entropy carbides

\begin{tabular}{|c|c|c|c|c|c|c|c|c|c|c|}
\hline \multirow[b]{2}{*}{ System } & \multicolumn{2}{|l|}{ B } & \multicolumn{2}{|l|}{$\boldsymbol{G}$} & \multicolumn{2}{|l|}{$\boldsymbol{E}$} & \multirow{2}{*}{$\begin{array}{l}H_{\mathrm{v}, \text { Chen }} \\
\text { AFLOW } \\
\text { (ROM) }\end{array}$} & \multirow{2}{*}{$\begin{array}{l}H_{v, \text { Teter }} \\
\text { AFLOW } \\
\text { (ROM) }\end{array}$} & \multirow{2}{*}{$\begin{array}{l}H_{\mathrm{v}, \text { Tian }} \\
\text { AFLOW } \\
\text { (ROM) }\end{array}$} & \multirow{2}{*}{$\begin{array}{l}H_{\mathrm{v}, \exp } \\
\text { Exp. } \\
\text { (ROM) }\end{array}$} \\
\hline & $\begin{array}{l}\text { AFLOW } \\
\text { (ROM) }\end{array}$ & $\begin{array}{l}\text { Exp. } \\
\text { (ROM) }\end{array}$ & $\begin{array}{l}\text { AFLOW } \\
\text { (ROM) }\end{array}$ & $\begin{array}{l}\text { Exp. } \\
\text { (ROM) }\end{array}$ & $\begin{array}{l}\text { AFLOW } \\
\text { (ROM) }\end{array}$ & Exp. (ROM) & & & & \\
\hline $\mathrm{MoNbTaVWC}_{5}$ & 312 (321) & $278(-)$ & $183(183)$ & $226(-)$ & $460(459)$ & $533 \pm 32(-)$ & $20(20)$ & $28(28)$ & $20(20)$ & $27 \pm 3(-)$ \\
\hline $\mathrm{HfNbTaTiZrC}_{5}$ & $262(267)$ & $\begin{array}{l}235 \\
(232)\end{array}$ & $192(165)$ & $\begin{array}{l}188 \\
(184)\end{array}$ & $464(455)$ & $\begin{array}{l}443 \pm 40 \\
(436 \pm 30)\end{array}$ & $27(25)$ & $29(28)$ & $27(25)$ & $\begin{array}{l}32 \pm 2 \\
(23 \pm 2)\end{array}$ \\
\hline HfNbTaTiVC $_{5}$ & 276 (279) & $\begin{array}{l}267 \\
(239)\end{array}$ & 196 (196) & $\begin{array}{l}212 \\
(189)\end{array}$ & $475(476)$ & $\begin{array}{l}503 \pm 40 \\
(449 \pm 30)\end{array}$ & $26(26)$ & $30(30)$ & $26(26)$ & $\begin{array}{l}29 \pm 3 \\
(24 \pm 2)\end{array}$ \\
\hline $\mathrm{HfNbTaTiWC}_{5}$ & 291 (296) & $252(-)$ & $203(186)$ & $205(-)$ & 493 (459) & $483 \pm 24(-)$ & $26(22)$ & $31(2 \varepsilon$ & $26(22)$ & $31 \pm 2(-)$ \\
\hline $\mathrm{NbTaTiVWC}_{5}$ & 305 (304) & $253(-)$ & 199 (189) & $206(-)$ & $490(460)$ & $485 \pm 36(-)$ & $24(22)$ & $30(29)$ & $24(23)$ & $28 \pm 2(-)$ \\
\hline HfTaTiWZrC $_{5}$ & $274(280)$ & $246(-)$ & $191(178)$ & $200(-)$ & $466(438)$ & $473 \pm 26(-)$ & $25(21)$ & $29(27)$ & $25(21)$ & $33 \pm 2(-)$ \\
\hline
\end{tabular}

Three different models: Chen et al, 54 Teter 55 , and Tian et al. 56 are used to calculate theoretical hardness values (only for the two non-W-containing compositions) from $B$ and $G$. The ROM values are obtained from the results in this work for rock-salt structure binary carbides, as listed in Table 3 . Since MoC and WC do not form a stable rock-salt phase at room temperature, the experimental ROMs for Mo- and W-containing compositions are not available, as indicated by (-). Units: $B, G, E$, and $H_{v}$ in (GPa)

Table 3 Results for mechanical properties (bulk: $B$, shear: $G$, and elastic: $E$, moduli, and Vickers hardness: $H_{\mathbf{V}}$ ) for eight rock-salt structure binary carbides

\begin{tabular}{|c|c|c|c|c|c|c|c|c|c|c|c|c|c|c|}
\hline \multirow[b]{2}{*}{ System } & \multicolumn{3}{|l|}{ B } & \multicolumn{3}{|l|}{ G } & \multicolumn{3}{|l|}{$\boldsymbol{E}$} & \multicolumn{5}{|l|}{$H_{v}$} \\
\hline & AFLOW & Exp. & Exp. $^{a}$ & AFLOW & Exp. & Exp. $^{a}$ & AFLOW & Exp. & Exp. ${ }^{a}$ & Chen & Teter & Tian & Exp. & Exp. $^{a}$ \\
\hline $\mathrm{HfC}$ & 239 & 241 & 223 & 186 & $179-193$ & 181 & 443 & $316-461$ & $428 \pm 32$ & 29 & 28 & 28 & $19-25$ & $25 \pm 2$ \\
\hline MoC & 335 & - & - & 152 & - & - & 396 & - & - & 12 & 23 & 13 & $27-83^{b}$ & - \\
\hline $\mathrm{NbC}$ & 297 & $296-378$ & 246 & 199 & $197-245$ & 177 & 488 & $330-537$ & $429 \pm 46$ & 25 & 30 & 25 & $19-25$ & $17 \pm 3$ \\
\hline $\mathrm{TaC}$ & 326 & $248-343$ & 219 & 213 & $215-227$ & 184 & 525 & $241-722$ & $431 \pm 44$ & 25 & 32 & 25 & $16-23$ & $14 \pm 2$ \\
\hline $\mathrm{TiC}$ & 251 & 241 & 255 & 181 & 186 & 207 & 438 & $447-451$ & $489 \pm 13$ & 26 & 27 & 25 & 32 & $31 \pm 2$ \\
\hline VC & 283 & 389 & 250 & 199 & 157 & 196 & 484 & $268-420$ & $465 \pm 13$ & 26 & 30 & 26 & $20-29$ & $29 \pm 1$ \\
\hline WC & 365 & - & - & 153 & - & - & 403 & - & - & 11 & 23 & 12 & $>28^{c}$ & - \\
\hline $\mathrm{ZrC}$ & 221 & 220 & 216 & 157 & 172 & 169 & 381 & $385-406$ & $402 \pm 13$ & 23 & 28 & 22 & $23-25$ & $24 \pm 1$ \\
\hline
\end{tabular}

The AFLOW values are calculated using the Voigt-Reuss-Hill average and the Automatic Elasticity Library (AEL) module ${ }^{53}$, whereas $H_{V}$ is estimated using three different models described in the literature. These results are compared with two sets of available measured data, obtained from the literature ${ }^{72,73}$ and the current experiments. Units: $B, G, E$, and $H_{\vee}$ in (GPa)

aThis work



${ }^{c} \mathrm{WC}_{1-x ;} ; x=0.36-0.4175$

Elastic properties are calculated using AFLOW ${ }^{41,53}$ for the fivemetal compositions MoNbTaVWC , HfNbTaTiZrC $_{5}$, HfNbTa$\mathrm{TiVC}_{5}, \mathrm{HfNbTaTiWC}_{5}, \mathrm{NbTaTiVWC}_{5}$, and $\mathrm{HfTaTiWZrC}_{5}$ (Table 2) and their precursors. In general, results are within the experimentally reported ranges for the binary carbides (Table 3 in the Methods section). $H_{\mathrm{V}}$ values are estimated from the bulk and shear moduli using the models introduced by Chen et al. ${ }^{54}$, Teter ${ }^{55}$, and Tian et al. ${ }^{56}$. Computational models do not consider plastic deformation mechanisms in inhomogeneous systems and thus $H_{\mathrm{V}}$ predictions underestimate experiments, leading to results consistent with the ROM of binary carbides (Table 2). The outcome further corroborates that the experimentally observed enhancement of the mechanical properties is due to disorder.

Vibrational contribution to formation free energy. The vibrational contributions to the formation Gibbs free energy, $\Delta F_{\text {vib }}$, at $2000 \mathrm{~K}$ are listed in Table 1 for the six compositions synthesized as a single phase. The vibrational free energies, $F_{\mathrm{vib}}$, are calculated using the Debye-Grüneisen model implemented in the AFLOW-Automatic GIBBS Library (AGL) module ${ }^{57}$, using the Poisson ratio calculated with AFLOW-Automatic Elasticity Library (AEL) ${ }^{53}$. The average $F_{\text {vib }}$ for the five-metal compositions are calculated, weighted according to the Boltzmann distribution at $2000 \mathrm{~K}$. The vibrational contribution to the formation Gibbs free energy, $\Delta F_{\text {vib }}$, for each composition is obtained from the difference between its average $F_{\text {vib }}$ and the average $F_{\text {vib }}$ of its component binary carbides. $\Delta F_{\text {vib }}$ at $2000 \mathrm{~K}$ ranges from $\sim 0 \mathrm{meV} /$ atom for $\mathrm{HfNbTaTiWC}_{5}$ to $-31 \mathrm{meV} /$ atom for $\mathrm{HfNbTaTiVC}_{5}$, which are significantly less than the total entropy contribution (mostly configurational plus vibrational) required to overcome the values of $50 \mathrm{meV} /$ atom to $150 \mathrm{meV} /$ atom for the formation enthalpy $\Delta H_{\mathrm{f}}$. These results are in agreement with previous observations that the vibrational formation entropy is generally an order of magnitude smaller than the configuration entropy ${ }^{39,58}$.

\section{Discussion}

In this article, an EFA descriptor has been developed for the purpose of capturing synthesizability of high-entropy materials. The framework has been applied to refractory metal carbides, leading to the prediction and subsequent experimental discovery of homogeneous high-entropy single phases. The method is able to quantitatively predict the relative propensity of each composition to form a homogeneous single phase, thus identifying the most promising candidates for experimental synthesis. In particular, the experiments validate the prediction that the composition $\mathrm{MoNbTaVWC}_{5}$ should have a very high propensity to form a homogeneous single phase, despite incorporating both $\mathrm{Mo}_{2} \mathrm{C}$ and $\mathrm{W}_{2} \mathrm{C}$, which have different structures (hexagonal and/or orthorhombic instead of rock-salt) and stoichiometric ratios from the five-metal high-entropy material. 
Furthermore, it is demonstrated that disorder enhances the mechanical properties of these materials: $\mathrm{HfNbTaTiZrC}_{5}$ and $\mathrm{HfTaTiWZrC}_{5}$ are measured to have hardness of $32 \mathrm{GPa}$ (almost $50 \%$ higher than the ROM prediction) and $33 \mathrm{GPa}$, respectively, suggesting a new avenue for designing super-hard materials. The formalism could become the long-sought enabler of accelerated design for high-entropy functional materials with enhanced properties for a wide range of different technological applications.

\section{Methods}

Spectrum generation. The different possible configurations required to calculate the energy spectrum are generated using the AFLOW-POCC algorithm ${ }^{41}$ implemented within the AFLOW computational materials design framework ${ }^{51,59,60}$. The algorithm initially generates a superlattice of the minimum size necessary to obtain the required partial occupancies within some user-specified accuracy. For each unique superlattice, the AFLOW-POCC algorithm then generates the complete set of possible supercells using Hermite normal form matrices ${ }^{41}$. Non-unique supercell combinations are eliminated from the ensemble by first estimating the total energies of all configurations using a Universal Force Field ${ }^{41,61}$ based method, and then identifying duplicates from their identical energies.

Structure generation. In the case of the high-entropy carbide ${ }^{17}$ systems investigated here, the AFLOW-POCC algorithm starts with the rock-salt crystal structure (spacegroup: $F m \overline{3} m$, \#225; Pearson symbol: cF8; AFLOW Prototype: AB_cF8_225_a_b ${ }^{62}$ ) as the input parent lattice. Each anion site is occupied with a $\mathrm{C}$ atom (occupancy probability of 1.0), whereas the cation site is occupied by five different refractory metal elements, with a 0.2 occupancy probability for each. The AFLOW-POCC algorithm then generates a set of configurations (49 in total in the case of the rock-salt based five-metal carbide systems, once structural duplicates are excluded), each containing 10 atoms: one atom of each of the metals, along with five carbon atoms. This is the minimum cell size necessary to accurately reproduce the required stoichiometry. All configurations have $g_{i}=10$, except for one where $g_{i}$ $=120$, so that $\sum_{i=1}^{n} g_{i}=600$ for the rock-salt-based five-metal carbide systems. Note that computational demands increase significantly with the number of elements: AFLOW-POCC generates 522, 1793, and 7483 for six-, seven-, and eightmetal carbide compositions, respectively.

Energies calculation. The energy of each configuration is calculated using density functional theory (Vienna Ab-initio Simulation Package ${ }^{63}$ ) within the AFLOW framework ${ }^{59}$ and the standard settings ${ }^{60}$. Each configuration is fully relaxed using the Perdew, Burke, Ernzerhof (PBE) parameterization of the generalized gradient approximation exchange-correlation functional ${ }^{64}$, projector augmented wave potentials, at least $8000 \mathbf{k}$-points per reciprocal atom (KPPRA), and a plane-wave cut-off of at least 1.4 times the cut-off values of constituent species' pseudopotentials ${ }^{60}$. The formation enthalpy $\left(H_{\mathrm{f}}\right)$ of each configuration along with the link to AFLOW.org entry page is provided in Supplementary Tables 4-10.

Mechanical properties. Elastic properties are calculated using the AEL module ${ }^{53}$ of the AFLOW framework, which applies a set of independent directional normal and shear strains to the structure, and fits the resulting stress tensors to obtain the elastic constants. From this, the bulk: $B$, and shear: $G$, moduli are calculated in the Voigt, Reuss and Voigt-Reuss-Hill (VRH) approximations, with the average being used for the purposes of this work. The elastic or Young's modulus: $E$, is calculated using the approximation $E=9 B G /(3 B+G)$, which can be derived starting from the expression for Hooke's Law in terms of $E$ and the Poisson ratio, $v: \varepsilon_{11}=1 / E\left[\sigma_{11}-v\right.$ $\left.\left(\sigma_{22}+\sigma_{33}\right)\right]^{65}$, and similarly for $\varepsilon_{22}$ and $\varepsilon_{33}$. For a cubic system, $\varepsilon_{11}=S_{11} \sigma_{11}+$ $S_{12} \sigma_{22}+S_{12} \sigma_{33}$ (similarly for $\varepsilon_{22}$ and $\varepsilon_{33}$ ), where $S_{i j}$ are the elements of the elastic compliance tensor, so that $1 / E=S_{11}$ and $-v / E=S_{12}$. For a cubic system, the bulk modulus is $B=1 /\left[3\left(S_{11}+2 S_{12}\right)\right]=E /[3(1-2 v)]$. The Poisson ratio can be written as $v=(3 B-2 G) /(6 B+2 G)$, and combining with the expression for $B$ and rearranging gives the required $E=9 B G /(3 B+G)$.

The elastic properties for the five-metal compositions are first calculated for each of the 49 configurations generated by AFLOW-POCC. The VRH approximated values of $B$ and $G$ for these configurations are listed in Supplementary Table 3, along with the AFLOW-POCC ensemble averaged electronic density of states (see Supplementary Figure 39). The average elastic moduli are then obtained, weighted according to the Boltzmann distribution at a temperature of $2200{ }^{\circ} \mathrm{C}$ (the experimental sintering temperature). These calculated values are compared with those obtained using a ROM (average of the binary components, weighted according to fractional composition in the sample).

Three different models are used for predicting the Vickers hardness based on the elastic moduli: Chen et al. $\left(H_{\mathrm{V}}=2\left(k^{2} G\right)^{0.585}-3 ; k=G / B\right)^{54}$, Teter $\left(H_{\mathrm{V}}=\right.$ $0.151 G)^{55}$, and Tian et al. $\left(H_{\mathrm{V}}=0.92 k^{1.137} G^{0.708} ; k=G / B\right)^{56}$. Note, however, that these models are based on the elastic response of the materials, and do not take into account phenomena such as plastic deformation, slip planes, and lattice defects.
Sample preparation. Initial powders of each of the eight binary precursor carbides ( $\mathrm{HfC}, \mathrm{NbC}, \mathrm{TaC}, \mathrm{TiC}, \mathrm{Mo}_{2} \mathrm{C}, \mathrm{VC}, \mathrm{W}_{2} \mathrm{C}, \mathrm{ZrC}$ ) are obtained in $>99 \%$ purity and -325 mesh $(<44 \mu \mathrm{m})$ particle size (Alfa Aesar). Samples are weighed out in $15 \mathrm{~g}$ batches and mixed to achieve the desired five-metal carbide compositions. To ensure adequate mixing, each sample is ball milled in a shaker pot mill for a total of $2 \mathrm{~h}$ in individual 30-min intervals intersected by 10 -min rest times to avoid heating and consequent oxide formation. All milling is done in tungsten carbide-lined stainless steel milling jars with tungsten carbide grinding media.

Bulk sample pellets are synthesized via solid-state processing routes. The fieldassisted sintering technique (FAST), also called SPS, is employed to simultaneously densify and react the compositions into single-phase materials. For all samples, sintering is done at $2200^{\circ} \mathrm{C}$ with a heating rate of $100^{\circ} \mathrm{C} / \mathrm{min}, 30 \mathrm{MPa}$ uniaxial pressure, and a 5-min dwell at temperature. Samples are heated in vacuum atmosphere to $1300^{\circ} \mathrm{C}$ followed by flowing argon to $2200^{\circ} \mathrm{C}$. All sintering is done in $20 \mathrm{~mm}$ graphite die and plunger sets with graphite foil surrounding the samples to prevent reaction with the die.

Sample analysis. Elemental analysis is performed using an FEI Quanta 600 SEM equipped with a Bruker e-Flash EDS detector at an accelerating voltage of $20 \mathrm{kV}$. Microstructural scanning electron microscope (SEM) imaging is carried out using an FEI Apreo FE-SEM at an accelerating voltage of $5 \mathrm{kV}$, with a combination of secondary and back-scattered electron detectors to show phase contrast. Crystal phase analysis is performed using a Rigaku Miniflex X-ray Diffractometer with a stepsize of $0.02^{\circ}$ and 5-s dwells, using $\mathrm{Cu} \mathrm{K} \alpha$ radiation (wavelength $\lambda=1.54059 \AA$ ) for all measurements and calculation of the lattice parameter. All sample patterns are fitted in Materials Data Incorporated's (MDI) Jade 9 software ${ }^{66}$ with a residual of fit $R<8 \%$. Lattice parameter, $a_{\text {exp }}$, values of $4.353 \AA$, $4.500 \AA$, $4.415 \AA, 4.434 \AA$, $4.355 \AA$, $4.502 \AA, 4.506 \AA$, $4.534 \AA$, and $4.476 \AA$ were measured for MoNbTaVWC ${ }_{5}$, HfNbTaTiZrC $_{5}$, HfNbTaTiVC $_{5}$, HfNbTaTiWC $_{5}$, NbTaTiVWC $_{5}$, HfTaTiWZrC $_{5}$, HfMoTaWZrC ${ }_{5}, \mathrm{HfMoVWZrC}_{5}$, and HfMoTiWZrC ${ }_{5}$, respectively (for multiphase samples, $a_{\exp }$ refers to the primary cubic phase).

For analysis of sample peak broadening $\beta_{\mathrm{S}}$, instrumental broadening $\beta_{\mathrm{I}}$ must first be determined. For this, a NIST $660 \mathrm{~b} \mathrm{LaB}_{6}$ standard is run under the same conditions as each carbide sample. The instrumental profile is then fitted, and $\beta_{\mathrm{I}}$ is determined to vary with Bragg angle $\theta$ as:

$$
\beta_{\mathrm{I}}=0.1750985-0.001560626 \theta+0.00001125342 \theta^{2} .
$$

$\beta_{\mathrm{S}}$ is determined by subtracting $\beta_{\mathrm{I}}$ from the measured broadening $\beta_{\mathrm{M}}$ : $\beta_{\mathrm{S}}^{x}=\beta_{\mathrm{M}}^{x}-\beta_{I}^{x}$. $\beta_{\mathrm{M}}$ is measured as a function of $\theta$, and $x$ is a constant between 1.0 and 2.0. In the current analysis, $x$ is set to 2.0 due to the Gaussian-like shape of the instrument peaks, as this value leads to the lowest standard deviation of linear fits to the peak broadening data.

Both crystallite size and lattice strain contribute to $\beta_{\mathrm{S}}{ }^{49,67,68:}$

$$
\beta_{\mathrm{S}}=4 \varepsilon \tan \theta+\frac{K \lambda}{D \cos \theta},
$$

where $\varepsilon$ is the lattice strain or variation in interplanar spacing due to chemical inhomogeneity, $K$ is a constant (dependent on the grain shape), $\lambda$ is the incident $\mathrm{X}$ ray wavelength and $D$ is the crystallite size. Rearranging Eq. 6 gives:

$$
\beta_{\mathrm{S}} \cos \theta=4 \varepsilon \sin \theta+\frac{K \lambda}{D}
$$

The slope of a linear fit to the plot of $\beta_{\mathrm{S}} \cos \theta$ against $\sin \theta$ is equal to the strain, or lattice distortion, whereas the $y$-intercept of a linear fit with zero slope determines the crystallite size.

Mechanical testing. Mechanical properties of each of the single-phase compositions are tested using a Keysight NanoIndenter G200 with a Berkovich indenter tip. To rule out indentation size effects, testing is carried out at loads of both $50 \mathrm{mN}$ and $300 \mathrm{mN}$, and no significant deviation in hardness or modulus is observed. To allow valid cross-comparison, each of the high entropy carbides is compared with the binary carbides, which were hot-pressed and indentation tested under identical conditions. For the reported values, tests are carried out according to the standard method outlined in ISO 14577 using a maximum load of $50 \mathrm{mN}$. Values are calculated as an average of 40 indents, and are reported with errors of plus or minus one standard deviation. A fused crystal silica standard is run prior to each test to ensure proper equipment calibration is maintained. Samples are polycrystalline with grain sizes between $10 \mu \mathrm{m}$ and $30 \mu \mathrm{m}$. Prior to indentation testing each sample is vibratory polished using $0.05 \mu \mathrm{m}$ colloidal silica for $12 \mathrm{~h}$ to ensure minimal surface roughness. All tests are carried out at a temperature of $27^{\circ} \mathrm{C} \pm 0.5$ ${ }^{\circ} \mathrm{C}$. Indentation data are analyzed according to the methods of Oliver and Pharr ${ }^{69,70}$. The elastic (i.e., Young's) modulus is determined using $1 / E_{\text {eff }}=\left(1-\nu^{2}\right) / E+\left(1-\nu_{\mathrm{I}}^{2}\right) / E_{\mathrm{I}}$, where $E_{\text {eff }}$ is the effective modulus (sometimes called the reduced modulus) obtained from nanoindentation, $E$ and $v$ are the Young's modulus and Poisson's ratio, respectively, for the specimen, whereas $E_{\mathrm{I}}$ and $v_{\mathrm{I}}$ are the same parameters for the indenter. A Poisson's ratio for each of the binary carbides is obtained from literature $\mathrm{e}^{71}$ where available. For five-metal carbide 
samples where data for each of the constituents is available, the value used for Poisson's ratio is taken as the average of the constituent binaries. If this average is unavailable (i.e., when Mo and/or W are present), Poisson's ratio is assumed to be equal to 0.18 .

\section{Data availability}

All the ab-initio data are freely available to the public as part of the AFLOW online repository and can be accessed through AFLOW.org following the REST-API interface $^{59}$ and AFLUX search language ${ }^{76}$

Received: 29 June 2018 Accepted: 18 October 2018

Published online: 26 November 2018

\section{References}

1. Gao, M. C, Yeh, J. W, Liaw, P. K. \& Zhang, Y. High-Entropy Alloys: Fundamentals and Applications. (Springer, Cham, Switzerland, 2016).

2. Senkov, O. N., Miller, J. D., Miracle, D. B. \& Woodward, C. Accelerated exploration of multi-principal element alloys with solid solution phases. Nat Commun. 6, 6529 (2015)

3. Widom, M. Modeling the structure and thermodynamics of high-entropy alloys. J. Mater. Res. 33, 2881-2898 (2018).

4. Lim, X. Mixed-up metals make for stronger, tougher, stretchier alloys. Nature 533, 306-307 (2016).

5. Ye, Y. F., Wang, Q., Lu, J., Liu, C. T. \& Yang, Y. High-entropy alloy: challenges and prospects. Mater. Today 19, 349-362 (2016).

6. Gludovatz, B. et al. A fracture-resistant high-entropy alloy for cryogenic applications. Science 345, 1153-1158 (2014).

7. Gali, A. \& George, E. P. Tensile properties of high- and medium-entropy alloys. Intermetallics 39, 74-78 (2013).

8. Senkov, O. N., Wilks, G. B., Scott, J. M. \& Miracle, D. B. Mechanical properties of $\mathrm{Nb}_{25} \mathrm{Mo}_{25} \mathrm{Ta}_{25} \mathrm{~W}_{25}$ and $\mathrm{V}_{20} \mathrm{Nb}_{20} \mathrm{Mo}_{20} \mathrm{Ta}_{20} \mathrm{~W}_{20}$ refractory high entropy alloys. Intermetallics 19, 698-706 (2011).

9. Li, Z., Pradeep, K. G., Deng, Y., Raabe, D. \& Tasan, C. C. Metastable highentropy dual-phase alloys overcome the strength-ductility trade-off. Nature 534, 227-230 (2016).

10. Tsao, T.-K. et al. The high temperature tensile and creep behaviors of high entropy superalloy. Sci. Rep. 7, 12658 (2017).

11. Li, Z., Tasan, C. C., Springer, H., Gault, B. \& Raabe, D. Interstitial atoms enable joint twinning and transformation induced plasticity in strong and ductile high-entropy alloys. Sci. Rep. 7, 40704 (2017).

12. Senkov, O. N., Wilks, G. B., Miracle, D. B., Chuang, C. P. \& Liaw, P. K. Refractory high-entropy alloys. Intermetallics 18, 1758-1765 (2010).

13. Senkov, O. N., Senkova, S. V., Woodward, C. \& Miracle, D. B. Low-density, refractory multi-principal element alloys of the $\mathrm{Cr}-\mathrm{Nb}-\mathrm{Ti}-\mathrm{V}-\mathrm{Zr}$ system: microstructure and phase analysis. Acta Mater. 61, 1545-1557 (2013).

14. von Rohr, F., Winiarski, M. J., Tao, J., Klimczuk, T. \& Cava, R. J. Effect of electron count and chemical complexity in the Ta-Nb-Hf-Zr-Ti highentropy alloy superconductor. Proc. Natl Acad. Sci. USA 113, E7144-E7150 (2016).

15. Bérardan, D., Franger, S., Dragoe, D., Meena, A. K. \& Dragoe, N. Colossal dielectric constant in high entropy oxides. Phys. Status Solidi RRL 10, 328-333 (2016).

16. Bérardan, D., Franger, S., Meena, A. K. \& Dragoe, N. Room temperature lithium superionic conductivity in high entropy oxides. J. Mater. Chem. A 4, 9536-9541 (2016).

17. Rost, C. M. et al. Entropy-stabilized oxides. Nat Commun. 6, 8485 (2015).

18. Rak, Z. et al. Charge compensation and electrostatic transferability in three entropy-stabilized oxides: results from density functional theory calculations. J. Appl. Phys. 120, 095105 (2016).

19. Gild, J. et al. High-entropy metal diborides: a new class of high-entropy materials and a new type of ultrahigh temperature ceramics. Sci. Rep. 6, 37946 (2016).

20. Castle, E., Csanádi, T., Grasso, S., Dusza, J. \& Reece, M. Processing and properties of high-entropy ultra-high temperature carbides. Sci. Rep. 8, 8609 (2018).

21. Dusza, J. et al. Microstructure of (Hf-Ta-Zr-Nb)C high-entropy carbide at micro and nano/atomic level. J. Eur. Ceram. Soc. 38, 4303-4307 (2018).

22. Yan, $\mathrm{X}$. et al. $\left(\mathrm{Hf}_{0.2} \mathrm{Zr}_{0.2} \mathrm{Ta}_{0.2} \mathrm{Nb}_{0.2} \mathrm{Ti}_{0.2}\right) \mathrm{C}$ high-entropy ceramics with low thermal conductivity. J. Am. Ceram. Soc. 101, 4486-4491 (2018).

23. Zhou, J. et al. High-entropy carbide: a novel class of multicomponent ceramics. Ceramics Int. 44, 22014-22018 (2018).

24. Meisenheimer, P. B., Kratofil, T. J. \& Heron, J. T. Giant enhancement of exchange coupling in entropy-stabilized oxide heterostructures. Sci. Rep. 7, 13344 (2017).
25. Rohrer, G. S. et al. Challenges in ceramic science: a report from the workshop on emerging research areas in ceramic science. J. Am. Ceram. Soc. 95 , 3699-3712 (2012)

26. Agte, C. \& Alterthum, H. Untersuchungen über systeme hochschmelzender carbide nebst beiträgen zum problem der kohlenstoffschmelzung [Investigations of high-melting point carbide systems and their contribution to the problem of carbon fusion]. Z. Tech. Phys. 11, 182-191 (1930)

27. Andrievskii, R. A., Strel'nikova, N. S., Poltoratskii, N. I., Kharkhardin, E. D. \& Smirnov, V. S. Melting point in systems ZrC-HfC, TaC-ZrC, TaC-HfC. Poroshk. Metall. 1, 85-88 (1967).

28. Hong, Q.-J. \& van de Walle, A. Prediction of the material with highest known melting point from ab initio molecular dynamics calculations. Phys. Rev. B 92, 020104 (2015).

29. Wuchina, E., Opila, E., Opeka, M., Fahrenholtz, W. \& Talmy, I. UHTCs: ultra-high temperature ceramic materials for extreme environment applications. Electrochem. Soc. Interface 16, 30-36 (2007).

30. Rudy, E. Ternary Phase Equilibria in Transition Metal-Boron-Carbon-Silicon Systems. Part II. Ternary Systems. (Air Force Materials Laboratory, WrightPatterson Air Force Base, Ohio, USA, 1965).

31. Rudy, E. Ternary Phase Equilibria in Transition Metal-Boron-Carbon-Silicon Systems. Part V. Compendium of Phase Diagram Data. (Air Force Materials Laboratory, Wright-Patterson Air Force Base, Ohio, USA, 1969).

32. Gusev, A. I. Phase diagrams of the pseudo-binary TiC-NbC, TiC-TaC, ZrC$\mathrm{NbC}, \mathrm{ZrC}-\mathrm{TaC}$, and HfC-TaC carbide systems. Russ. J. Phys. Chem. 59, 336-340 (1985).

33. Cedillos-Barraza, O. et al. Sintering behaviour, solid solution formation and characterisation of TaC, HfC and TaC-HfC fabricated by spark plasma sintering. J. Eur. Ceram. Soc. 36, 1539-1548 (2016).

34. Cedillos-Barraza, $\mathrm{O}$. et al. Investigating the highest melting temperature materials: a laser melting study of the TaC-HfC system. Sci. Rep. 6, 37962 (2016).

35. Landau, D. P., Tsai, S.-H. \& Exler, M. A new approach to Monte Carlo simulations in statistical physics: Wang-Landau sampling. Am. J. Phys. 72, 1294-1302 (2004)

36. Baldock, R. J. N., Pártay, L. B., Bartók, A. P., Payne, M. C. \& Csányi, G. Determining pressure-temperature phase diagrams of materials. Phys. Rev. B 93, 174108 (2016)

37. Gao, M. C. \& Alman, D. E. Searching for next single-phase high-entropy alloy compositions. Entropy 15, 4504-4519 (2013).

38. Zhang, F. et al. An understanding of high entropy alloys from phase diagram calculations. Calphad 45, 1-10 (2014).

39. Gao, M. C. et al. Thermodynamics of concentrated solid solution alloys Curr. Opin. Solid State Mater. Sci. 21, 238-251 (2017).

40. Curtarolo, S. et al. The high-throughput highway to computational materials design. Nat. Mater. 12, 191-201 (2013).

41. Yang, K., Oses, C. \& Curtarolo, S. Modeling off-stoichiometry materials with a high-throughput ab-initio approach. Chem. Mater. 28, 6484-6492 (2016).

42. Bergerhoff, G., Hundt, R., Sievers, R. \& Brown, I. D. The inorganic crystal structure data base. J. Chem. Inf. Comput. Sci. 23, 66-69 (1983).

43. Massalski, T. B., Okamoto, H., Subramanian, P. R. \& Kacprzak, L. Binary Alloy Phase Diagrams. (ASM International, Materials Park, Ohio, USA, 1990)

44. Oses, C. et al. AFLOW-CHULL: cloud-oriented platform for autonomous phase stability analysis. J. Chem. Inf. Model. https://doi.org/10.1021/acs. jcim.8b00393 (2018)

45. Lederer, Y., Toher, C., Vecchio, K. S. \& Curtarolo, S. The search for high entropy alloys: a high-throughput ab-initio approach. Acta Mater. 159, 364-383 (2018).

46. Perim, E. et al. Spectral descriptors for bulk metallic glasses based on the thermodynamics of competing crystalline phases. Nat. Commun. 7, 12315 (2016).

47. Greer, A. L. Confusion by design. Nature 366, 303-304 (1993).

48. Kuo, K. \& Hägg, G. A new molybdenum carbide. Nature 170, 245-246 (1952)

49. Williamson, G. K. \& Hall, W. H. X-ray line broadening from filed aluminium and wolfram. Acta Metall. 1, 22-31 (1953).

50. Freudenberger, J. et al. Face centred cubic multi-component equiatomic solid solutions in the Au-Cu-Ni-Pd-Pt system. Metals 7, 135 (2017).

51. Curtarolo, S. et al. AFLOWLIB.ORG: a distributed materials properties repository from high-throughput $a b$ initio calculations. Comput. Mater. Sci. 58, 227-235 (2012)

52. Smith, C. J., Yu, X.-X., Guo, Q., Weinberger, C. R. \& Thompson, G. B. Phase, hardness, and deformation slip behavior in mixed $\mathrm{Hf}_{x} \mathrm{Ta}_{1-x} \mathrm{C}$. Acta Mater. 145, 142-153 (2018).

53. Toher, C. et al. Combining the AFLOW GIBBS and elastic libraries to efficiently and robustly screen thermomechanical properties of solids. Phys. Rev. Mater. 1, 015401 (2017).

54. Chen, X.-Q., Niu, H., Li, D. \& Li, Y. Modeling hardness of polycrystalline materials and bulk metallic glasses. Intermetallics 19, 1275-1281 (2011). 
55. Teter, D. M. Computational alchemy: the search for new superhard materials. MRS Bull. 23, 22-27 (1998).

56. Tian, Y., Xu, B. \& Zhao, Z. Microscopic theory of hardness and design of novel superhard crystals. Int. J. Refract. Met. Hard Mater. 33, 93-106 (2012).

57. Toher, C. et al. High-throughput computational screening of thermal conductivity, Debye temperature, and Grüneisen parameter using a quasiharmonic Debye model. Phys. Rev. B 90, 174107 (2014).

58. van de Walle, A. \& Ceder, G. The effect of lattice vibrations on substitutional alloy thermodynamics. Rev. Mod. Phys. 74, 11-45 (2002).

59. Curtarolo, S. et al. AFLOW: an automatic framework for high-throughput materials discovery. Comput. Mater. Sci. 58, 218-226 (2012).

60. Calderon, C. E. et al. The AFLOW standard for high-throughput materials science calculations. Comput. Mater. Sci. 108 Part A, 233-238 (2015).

61. Rappe, A. K., Casewit, C. J., Colwell, K. S., Goddard, W. A. III \& Skiff, W. M. UFF, a full periodic table force field for molecular mechanics and molecular dynamics simulations. J. Am. Chem. Soc. 114, 10024-10035 (1992).

62. Mehl, M. J. et al. The AFLOW library of crystallographic prototypes: part 1. Comput. Mater. Sci. 136, S1-S828 (2017).

63. Kresse, G. \& Furthmüller, J. Efficient iterative schemes for $a b$ initio total-energy calculations using a plane-wave basis set. Phys. Rev. B 54, 11169-11186 (1996).

64. Perdew, J. P., Burke, K. \& Ernzerhof, M. Generalized gradient approximation made simple. Phys. Rev. Lett. 77, 3865-3868 (1996).

65. Suh, N. P. \& Turner, A. P. L. Elements of the Mechanical Behavior of Solids (McGraw-Hill, New York, 1975).

66. Materials Data, Inc. Jade 9. https://materialsdata.com/prodjd.html (Materials Data Inc., 2016).

67. Cullity, B. D. Elements of X-Ray Diffraction. (Addison-Wesley, Reading, MA, USA, 1956).

68. Mote, V. D., Purushotham, Y. \& Dole, B. N. Williamson-Hall analysis in estimation of lattice strain in nanometer-sized $\mathrm{ZnO}$ particles. J. Theor. Appl. Phys. 6, 6 (2012).

69. Oliver, W. C. \& Pharr, G. M. An improved technique for determining hardness and elastic modulus using load and displacement sensing indentation experiments. J. Mater. Res. 7, 1564-1583 (1992).

70. Oliver, W. C. \& Pharr, G. M. Measurement of hardness and elastic modulus by instrumented indentation: advances in understanding and refinements to methodology. J. Mater. Res. 19, 3-20 (2004).

71. Kral, C., Lengauer, W., Rafaja, D. \& Ettmayer, P. Critical review on the elastic properties of transition metal carbides, nitrides and carbonitrides. J. Alloy. Compd. 265, 215-233 (1998).

72. American Society for Metals. ASM Engineered Materials Reference Book (ASM International, Materials Park, Ohio, USA, 1989).

73. Oyama, S. T. (ed.) The Chemistry of Transition Metal Carbides and Nitrides (Blackie Academic \& Professional, Glasgow, UK, 1996).

74. Dubitsky, G. A. et al. Superhard superconductor composites obtained by sintering of diamond, $c$ - $\mathrm{BN}$ and $\mathrm{C}_{60}$ powders with superconductors. $Z$. Naturforsch. B 61, 1541-1546 (2006).

75. Caron, P. \& Tremblay, A. Method for treating tungsten carbide particles. US patent 7981394 B2 (2011).

76. Rose, F. et al. AFLUX: The LUX materials search API for the AFLOW data repositories. Comput. Mater. Sci. 137, 362-370 (2017).

\section{Acknowledgements}

The authors acknowledge support by DOD-ONR (N00014-15-1-2863, N00014-17-12090, N00014-16-1-2583, N00014-17-1-2876) and by Duke University-Center for Materials Genomics-for computational support. S.C. acknowledges the Alexander von Humboldt Foundation for financial support. C.O. acknowledges support from the National Science Foundation Graduate Research Fellowship under grant no. DGF1106401. The authors thank Axel van de Walle, Matthias Scheffler, Claudia Draxl, Ohad Levy, Yoav Lederer, Amir Natan, Omar Cedillos Barraza, Joshua Gild, Olivia Dippo, and Cameron McElfresh for helpful discussions.

\section{Author contributions}

S.C. proposed the entropy spectral descriptor and the phase stabilization mechanism C.T. wrote the AEL-AGL codes under the supervision of S.C. C.O. wrote the AFLOWPOCC and AFLOW-CHULL codes under the supervision of C.T. and S.C. P.S. and C.T. performed the AFLOW-POCC and AEL-AGL calculations for the single-phase systems. C.O. performed the convex hull analysis. T.H. made the samples under the supervision of K.V. T.H. and M.S. performed the experimental characterization. All authors-P.S., T.H., C.T., C.O., M.S., J.-P.M., D.B., K.V., S.C.-discussed the results and contributed to the writing of the article.

\section{Additional information}

Supplementary Information accompanies this paper at https://doi.org/10.1038/s41467018-07160-7.

Competing interests: The authors declare no competing interests.

Reprints and permission information is available online at http://npg.nature.com/ reprintsandpermissions/

Publisher's note: Springer Nature remains neutral with regard to jurisdictional claims in published maps and institutional affiliations.

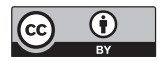

Open Access This article is licensed under a Creative Commons Attribution 4.0 International License, which permits use, sharing, adaptation, distribution and reproduction in any medium or format, as long as you give appropriate credit to the original author(s) and the source, provide a link to the Creative Commons license, and indicate if changes were made. The images or other third party material in this article are included in the article's Creative Commons license, unless indicated otherwise in a credit line to the material. If material is not included in the article's Creative Commons license and your intended use is not permitted by statutory regulation or exceeds the permitted use, you will need to obtain permission directly from the copyright holder. To view a copy of this license, visit http://creativecommons.org/ licenses/by/4.0/.

(C) The Author(s) 2018 